\title{
Interaction of Chemotherapy and Radiotherapy in Altering the Morphology of Subcortical Structures in Patients With Nasopharyngeal Carcinoma
}

\section{Feibiao Nan}

UESTC: University of Electronic Science and Technology of China

\section{You-ming Zhang}

Xiangya Hospital Central South University

Jian-ming Gao

Sun Yat-Sen University Cancer Center Department of Radiotherapy

Li Li

Sun Yat-sen University Cancer Center

Yuanchao Zhang ( $\nabla$ yuanchao.zhang8@gmail.com )

UESTC: University of Electronic Science and Technology of China https://orcid.org/0000-0001-81914899

Wei-hua Liao

Xiangya Hospital Central South University

\section{Research Article}

Keywords: Nasopharyngeal carcinoma, Subcortical structures, Radiotherapy, Chemoradiotherapy, Shape analysis

Posted Date: June 9th, 2021

DOl: https://doi.org/10.21203/rs.3.rs-556001/v1

License: (a) (1) This work is licensed under a Creative Commons Attribution 4.0 International License. Read Full License 


\section{Abstract}

Neuroimaging studies have found significant structural alterations of the cerebral cortex in patients with nasopharyngeal carcinoma (NPC) following radiotherapy (RT) or concomitant chemoradiotherapy (CCRT), while their effects on the morphology of subcortical structures remain largely unknown. In this study, we investigated the subcortical morphological alterations between three groups: 56 untreated NPC patients (pre-RT group), 37 RT-treated NPC patients (post-RT group), and 108 CCRT-treated NPC patients (post-CCRT group). Using FSL-FIRST, we found that, compared with the pre-RT group, the post-CCRT group exhibited morphological atrophy in the bilateral thalamus, bilateral putamen, left pallidum, and left caudate and morphological inflation in the left caudate, while the post-RT group only exhibited morphological atrophy in the bilateral thalamus. We also found a significant negative correlation between the maximum dosage of RT for temporal lobes and the morphological changes of the bilateral thalamus in treated NPC patients. These results indicated that there may be an interaction between RT and CT that can cause subcortical damage.

\section{Introduction}

Nasopharyngeal carcinoma (NPC) is an uncommon cancer arising from the nasopharynx epithelium. It is an endemic disease with a high incidence rate in east and southeast Asia (especially in southern China) (L. L. Tang et al., 2016). To date, radiotherapy (RT) has been considered the primary treatment modality for NPC(Chua, Wee, Hui, \& Chan, 2016). For patients with locally advanced NPC, chemotherapy (CT) can be applied alongside with RT. Despite the effectiveness of RT and CT in disease control(Blanchard et al., 2015; Yan, Kumachev, Siu, \& Chan, 2015), they are often associated with serious adverse effect on the central nervous system, leading to visual and auditory impairment, progressive memory loss, and increased negative emotions (depression, anxiety)(Hong, Tian, Han, \& Ni, 2015; Makale, McDonald, Hattangadi-Gluth, \& Kesari, 2017; Y. Tang, Luo, Rong, Shi, \& Peng, 2012). Moreover, it has been shown that concomitant chemo-radiotherapy (CCRT) is associated with more severe side effects on the brain than RT alone(Wang et al., 2019) or CT alone(Simo et al., 2016).

In recent years, advances in neuroimaging techniques have provided new and exciting avenues for us to noninvasively characterize the macroscopic changes of brain parenchyma in vivo. In NPC, most of previous neuroimaging studies were conducted attempting to examine the effects of RT on brain structure and function. For example, studies using structural MRI data have revealed that compared with patients before RT, patients with NPC have significantly decreased gray matter after RT, mainly involving the temporal lobe, hippocampus and the cerebellum, and that the gray matter decreases were dose-and time-dependent (Blanchard et al., 2015; Guo et al., 2018; Leng et al., 2017; Lin et al., 2017; Lv et al., 2014; Shi et al., 2018). Using diffusion tensor imaging (DTI), studies have found that RT could decrease fractional anisotropy and increase mean diffusivity of the white matter in cerebellum, temporal lobe, frontal lobe and parietal lobe in patients with NPC (Duan et al., 2016; Leng et al., 2017; Leng et al., 2019). A DTI-based network study found that brain global network properties (e.g. clustering coefficient, normalized characteristic path length, normalized clustering coefficient) decreased in patients with NPC 
within 6 months after RT, compared with those without RT (Q. Chen et al., 2020). Using resting-state fMRI, some studies have found that the cerebellum and some regions of default mode network, including the hippocampus, temporal lobe, and posterior cingulate cortex (PCC), were associated with altered functional connectivity to other regions in (S. C. Chen et al., 2017; Ding et al., 2018; W. T. Ren et al., 2019). Compared with patients with NPC who did not receive RT, RT-treated NPC patients had aberrant regional homogeneity (ReHo) values in cerebellum, temporal lobe and insula and aberrant ReHo values had different dynamic changes patterns over time (Yang et al., 2019). In contrast, less attention has been paid to the effect of CT on the brain. Using multimodal MRI data, our recent study investigated the effects of RT and CT on the cortical morphology and functional connectivity in patients with NPC, and found that chemotherapy potentially facilitated the occurrence of radiation encephalopathy in treated NPC patients (Y. Zhang et al., 2019). One question remains, however, as to whether RT and CT could affect the morphology of subcortical structures in patients with NPC.

In the present study, we aimed to investigate the effects of CT and RT on the morphology of subcortical structures in patients with NPC. Specifically, we used structural MRI data to characterize the shape abnormalities of subcortical nuclei in a cohort of patients with NPC who had undergone RT (Post-RT) or CCRT (Post-CCRT), as compared to untreated NPC patients (Pre-RT). We hypothesized that, compared with Pre-RT group, Post-RT group would show significant abnormalities in the shape of the subcortical nuclei including the thalamus, while Post-CCRT group would show more extensive changes in the shape of the subcortical nuclei including the thalamus.

\section{Materials And Methods}

\section{Subjects}

This retrospective cross-sectional study included 201 patients with pathologically diagnosed NPC. They were divided into three groups according to the difference in treatment methods: the Pre-RT group (56 NPC patients who had not received treatment), the Post-RT group (37 NPC patients who had received RT) and the Post-CCRT group (108 NPC patients who had received CCRT)(Y. Zhang et al., 2019). To measure the effect of RT or CCRT on the structure of subcortical nuclei, other possible confounding factors (such as age, sex, time intervals between RT and sMRI examinations, RT technology, and maximum RT dosage to the temporal lobes) had to be evenly distributed among the groups. The inclusion criteria for all patients in this study were as follows: (1) Pathologically confirmed NPC patients; (2) normal-appearing brain parenchyma on MRl; (3) right-handedness; (4) more than 6 years of education; and (5) an age range from 20 to 60 years. The exclusion criteria for all patients in this study were as follows: (1) brain parenchymal invasion, (2) brain tumor, (3) prior substantial head trauma or surgery, (4) neurological or psychiatric illness, (5) alcoholism or drug abuse, (6) any other major intracranial disease(Zhang et al., 2018).

For each NPC patient, the following clinical data were available: the Karnofsky Performance Status (KPS) score, the main side of NPC, clinical stage, RT techniques, maximum dosage of RT to the temporal lobes, 
time intervals between RT and MRI examination, detailed information on the chemotherapy agents. All patients receiving RT were given Intensity-modulated radiation therapy (IMRT) or conventional twodimensional radiotherapy (2D-CRT), as shown in Table 1. All patients presented with stage II-IV NPC according to the 7th edition of the UICC/AJCC (2009) TNM classification ( $\mathrm{T}=$ Tumor, $\mathrm{N}=$ Nodes, and $\mathrm{M}=$ Metastasis)(Edge \& Compton, 2010) and received CCRT, either with or without neoadjuvant/adjuvant chemotherapy. Specifically, the CCRT dosage regimen was cisplatin $\left(100 \mathrm{mg} / \mathrm{m}^{2}\right.$ intravenously on day 1$)$. Additional adjuvant chemotherapy regimen was 3 cycles of cisplatin $\left(80 \mathrm{mg} / \mathrm{m}^{2}\right.$ intravenously on day 1 ) and fluorouracil $\left(4 \mathrm{~g} / \mathrm{m}^{2}\right.$ in continuous intravenous infusion over $120 \mathrm{~h}$ ). Additional neoadjuvant chemotherapy regimen was two cycles of cisplatin $\left(80 \mathrm{mg} / \mathrm{m}^{2}\right.$ intravenously on day 1$)$ and fluorouracil (4 $\mathrm{g} / \mathrm{m}^{2}$ in continuous intravenous infusion over $120 \mathrm{~h}$ ). This study was approved by the Medical Research Ethics Committee of Xiangya Hospital, Central South University and performed in line with the principles of the Declaration of Helsinki and its later amendments. Written informed consent was obtained from all subjects. 
Table 1

Clinical data of the participants

\begin{tabular}{|c|c|c|c|c|}
\hline Items & pre-RT & post-RT & post-CCRT & p \\
\hline $\begin{array}{l}\text { Age (years), } \\
\text { (mean } \pm S D)\end{array}$ & $\begin{array}{l}47.0 \pm \\
9.0\end{array}$ & $\begin{array}{l}48.5 \pm \\
10.1\end{array}$ & $45.5 \pm 7.8$ & 0.17 \\
\hline \multicolumn{5}{|l|}{ Sex } \\
\hline male & $\begin{array}{l}43 \\
(21.4)\end{array}$ & $29(14.4)$ & $74(36.8)$ & \multirow[t]{2}{*}{0.59} \\
\hline female & $13(6.5)$ & $8(4.0)$ & $19(9.5)$ & \\
\hline \multicolumn{5}{|l|}{ Main side of NPC } \\
\hline Left, $n$ & $17(8.4)$ & $8(4.0)$ & $20(10.0)$ & \multirow[t]{3}{*}{0.51} \\
\hline Right, $n$ & $\begin{array}{l}22 \\
(10.9)\end{array}$ & $18(9.0)$ & $50(24.9)$ & \\
\hline Bilateral, n & $17(8.4)$ & $11(5.5)$ & $38(18.9)$ & \\
\hline \multicolumn{5}{|l|}{ Clinical staging } \\
\hline I/II, n & $14(7.0)$ & $29(14.4)$ & $23(11.4)$ & \multirow{2}{*}{$<.01 *$} \\
\hline III/IV, n & $\begin{array}{l}42 \\
(20.9)\end{array}$ & $8(4.0)$ & $85(42.3)$ & \\
\hline $\begin{array}{l}\text { Time intervals between RT and MRI examinations } \\
\text { (month) }\end{array}$ & NA & $\begin{array}{l}20.9 \pm \\
25.4\end{array}$ & $\begin{array}{l}16.9 \pm \\
23.8\end{array}$ & 0.39 \\
\hline \multicolumn{5}{|l|}{ RT technology } \\
\hline IMRT, n & NA & $28(19.3)$ & $86(59.3)$ & \multirow[t]{2}{*}{0.61} \\
\hline 2D-CRT, n & NA & $9(6.2)$ & $22(15.2)$ & \\
\hline \multirow[t]{2}{*}{ KPS score, (median \pm IQR, range) } & $90 \pm 0$ & $90 \pm 0$ & $90 \pm 0$ & \multirow[t]{2}{*}{0.59} \\
\hline & $80-90$ & $80-90$ & $80-90$ & \\
\hline \multicolumn{5}{|l|}{ Maximum dosage of RT for temporal lobes (Gy) } \\
\hline Left & NA & $\begin{array}{l}62.6 \pm \\
7.8^{\mathrm{a}}\end{array}$ & $\begin{array}{l}66.0 \pm \\
10.3^{b}\end{array}$ & 0.14 \\
\hline
\end{tabular}

SD, standard deviation; UICC, International Union against Cancer; AJCC, American Joint Committee on Cancer; RT, radiation therapy; IMRT, intensity-modulated radiation therapy; 2D-CRT, conventional twodimensional radiotherapy; KPS, Karnofsky Performance Status; IQR, interquartile range. (1) Data in parentheses are percentages; (2) The superscripts $a$ and $b$ denote data loss in 5 and 9 subjects respectively. ${ }^{*} \mathrm{P}<0.05$. 


\begin{tabular}{|c|c|c|c|c|}
\hline Items & pre-RT & post-RT & post-CCRT & p \\
\hline Right & NA & $\begin{array}{l}64.6 \pm \\
7.0^{\mathrm{a}}\end{array}$ & $67.5 \pm 8.1^{b}$ & 0.13 \\
\hline \multicolumn{5}{|c|}{$\begin{array}{l}\text { SD, standard deviation; UICC, International Union against Cancer; AJCC, American Joint Committee on } \\
\text { Cancer; RT, radiation therapy; IMRT, intensity-modulated radiation therapy; } 2 \mathrm{D}-\mathrm{CRT} \text {, conventional two- } \\
\text { dimensional radiotherapy; KPS, Karnofsky Performance Status; IQR, interquartile range. (1) Data in } \\
\text { parentheses are percentages; (2) The superscripts a and b denote data loss in } 5 \text { and } 9 \text { subjects } \\
\text { respectively. }{ }^{*} P<0.05 \text {. }\end{array}$} \\
\hline
\end{tabular}

\section{MRI Acquisitions}

All MRI data, including axial T1-weighted images, T2-weighted images, and T2-weighted fluid attenuated inversion recovery images for detecting any clinically silent lesions, were acquired on a Siemens Magnetom Tim Trio 3.0-T MR scanner with a 32-channel head coil. For each patient, high-resolution structural images were obtained using a T1-weighted 3D magnetization-prepared rapid acquisition gradient-echo sequence with 176 sagittal slices. The setting of scanning parameters in this study was as follows: repetition time $(T R)=2,300 \mathrm{~ms}$, echo time $(T E)=2.98 \mathrm{~ms}$, field of view $(F O V)=256 \times 256 \mathrm{~mm}$, matrix size $=256 \times 256$, thickness $/$ gap $=1.0 / 0 \mathrm{~mm}$, flip angle $=9^{\circ}$, and voxel size $=1.0 \times 1.0 \times 1.0 \mathrm{~mm}$. During scanning, each subject was asked to lie motionlessly, close eyes and remain as equanimous as possible.

\section{Data processing and statistical analyses}

All MRI data were analyzed with the software package FSL-FIRST (Analysis Group, FMRIB, Oxford, UK), a deformable-model-based active appearance model in a Bayesian framework(Patenaude, Smith, Kennedy, \& Jenkinson, 2011). Our images were automatically segmented by applying the manually labelled information in the training images to the model training. The specific process of constructing the model is as follows. First, linear subcortical registration was applied in all images to achieve the correct alignment between the model and images. Second, while maintaining cross-subject vertex correspondence, a deformable 3D mesh was used to fit the training images to obtain manually labelled information, and then iteratively update its vertex position. Third, appearance was modeled with normalized intensity, which was to sample each training intensity image along the surface normal of the mesh vertex. Finally, the variation in the mesh fitting process for each image was modelled by vertex coordinates and intensity samples. Before the new image was fitted with the model, the model was registered into the native space using inverse transformation, and then the optimal fitting was obtained by maximizing the posterior probability. In this way, for each subject, we can obtain a surface mesh consisting of vertices and triangles. Because the subcortical surface meshes of all subjects were isomorphic, the comparisons between groups could be performed by examining group differences in the spatial location of each vertex. Although the vertices were in correspondence, the surface mesh was not in standard space, but in its native space. Before investigating group differences, the pose differences were removed by rigid alignment of the mean surface mesh in standard space, which meant that the sum-of-squares between a subject's surface mesh and the mean surface mesh were minimized. After 
that, multivariate F-tests were performed for each vertex separately to detect between-group localized shape difference. Vertex-wise correlation analysis was also performed between the shape of subcortical nuclei and the maximum dosage of RT for temporal lobes in treated NPC patients. False discovery rate (FDR) theory was used to correct for multiple comparisons.

\section{Results}

Comparisons of the vertex locations between the Pre-RT group and the Post-RT group showed that PostRT group exhibited significant regional atrophy in the medial posterior segment of the bilateral thalamus (Fig. 1).

Similarly, compared with the Pre-RT group, the medial posterior segment of the bilateral putamen, bilateral thalamus, left pallidum, and the left caudate were substantially atrophied in the Post-CCRT group. Further, the Post-CCRT group showed a significant regional inflation in the lateral anterior segment of the left pallidum (Fig. 2).

In the post-treatment patient groups (including post-RT and post-CCRT groups), there was a significant negative correlation between the maximum dosage of RT for temporal lobes and the morphological changes of the superior segment of the bilateral thalamus (Fig. 3).

\section{Discussion}

In this study, we examined the subcortical shape abnormalities in patients with NPC who underwent RT or CCRT, compared with untreated NPC patients. Our main findings can be summarized as follows. First, compared with the pre-RT group, patients in the post-RT group showed significant regional atrophy in the medial posterior segment of the bilateral thalamus. Second, compared with the pre-RT group, patients in the post-CCRT group showed significant regional atrophy in the medial posterior segment of the bilateral thalamus, bilateral putamen, left pallidum, and left caudate, and significant regional inflation in the lateral anterior segment of the left pallidum. Finally, in treated NPC patients, the maximum dosage of RT for temporal lobes was negatively correlated with the morphological changes of the superior segment of the bilateral thalamus. Taken together, these findings suggest that RT and CT may interact in altering the morphology of subcortical structures in NPC.

Compared with the pre-RT group, patients in the post-RT group showed significant regional atrophy in the medial posterior segment of the bilateral thalamus. This finding is consistent with some previous fMRI studies, showing decreased fractional amplitude of low frequency fluctuation and ReHo values in the thalamus of NPC patients after RT(Ding et al., 2018; Y. M. Zhang et al., 2019). The observed regional atrophy in the bilateral thalamus might be the anatomical substrates of the functional alterations in NPC patients treated with RT. Compared with the pre-RT group, patients in the post-CCRT group showed significant atrophy in the medial posterior segment of bilateral thalamus. Of note, the thalamic area identified with significant regional atrophy in the post-CCRT group was much larger than that identified in 
the post-RT group, suggesting possible interactions between RT and CT. In addition, using the pooled data of the post-RT and post-CCRT groups, we also found a significant negative correlation between the morphological changes of the thalamus and the maximum dose of RT for temporal lobe, suggesting that the thalamic abnormalities could arise from the direct effects of RT or from abnormal afferent inputs from RT-lesioned temporal lobe via the reciprocal thalamo-cortical connections(Hwang, Bertolero, Liu, \& D'Esposito, 2017).

Compared with the pre-RT group, patients in the post-CCRT group showed significant atrophy in the medial posterior segment of the bilateral putamen, left pallidum, and the left caudate. This finding is consistent with previous neuroimaging studies conducted to investigate the effect of CT on the brain structure. For example, Nelson et al. found higher mean diffusivity values in multiple subcortical nuclei (thalamus, putamen, globus pallidus, etc.) in long-term survivors of childhood brain tumor after CT(Nelson et al., 2014). Similarly, two cross-sectional morphologic MRI studies showed significant reduction in the volume of multiple subcortical nuclei in long-term survivors of childhood acute lymphoblastic leukemia after CT(Genschaft et al., 2013; van der Plas et al., 2017). Compared with the preRT group, the present study also found significant regional inflation in the lateral anterior segment of the left pallidum in the post-CCRT group. The finding of concurrent atrophy and inflation in the pallidum may indicate functional heterogeneity of different nuclei of the pallidum. However, the underlying mechanism of such alteration remains unclear and requires further investigation. Collectively, given that no significant shape abnormalities in putamen, pallidum and caudate were found in the post-RT group, we therefore speculate that the shape alterations of these subcortical structures may primarily occur as a result of CT.

The exact pathophysiological mechanism that leads to these significantly morphological alterations of subcortical structures remains unclear. It is possible that multiple mechanisms, including DNA damage, apoptosis, inflammatory response and oxidative stress, vessel abnormity, and destruction of the bloodbrain barrier (BBB), are involved in the development of the brain injury after RT or CT(Lumniczky, Szatmari, \& Safrany, 2017; X. Ren et al., 2019). In fact, in the studies of the mechanism of RT or CT, the inflammatory response mediated by reactive microglia has always been of particular interest. More specifically, cellular debris from RT- or CT- damaged neuron could activate microglia, which in turn could release pro-inflammatory cytokines and other neurotoxic factors that damage neurons(Block, Zecca, \& Hong, 2007). Similarly, there is evidence that reactive astrocytes induced by reactive microglia are also neurotoxic(Liddelow et al., 2017). Besides, increased BBB permeability caused by CCRT also seems to be able to indirectly exacerbate existing levels of inflammatory response(Zlokovic, 2008). However, we cannot exclude the involvement of other mechanisms in causing the morphologic alterations in treated NPC patients.

Functionally, the thalamus, as a hub, plays a particularly critical role in controlling the transmission and integration of information flow in the cortical and subcortical networks, which is the basis for its support of related cognitive functions(Hwang et al., 2017; Nakajima \& Halassa, 2017; Sherman, 2016). The thalamus and its connections to other brain regions (including the medial temporal lobe, cerebellum, prefrontal cortex, etc.) are instrumental in several neurocognitive domains, including memory, executive 
function, sleep and arousal, and information processing speed (Bolkan et al., 2017; Hwang et al., 2017; Kafkas, Mayes, \& Montaldi, 2020; Nakajima \& Halassa, 2017; Niendam et al., 2012; Prevosto \& Sommer, 2013), which are thought to be most affected by RT or CT(Ahles \& Saykin, 2007; Lumniczky et al., 2017). Similarly, the putamen, the pallidum and the caudate have been involved in learning, language and reward mechanism, which are closely related to cognitive function(Bick et al., 2019; Doi, Fan, Gold, \& Ding, 2020; Hervais-Adelman, Egorova, \& Golestani, 2018; Vinas-Guasch \& Wu, 2017). The morphological alterations of these structures may be related to the neurocognitive changes after RT or CCRT for NPC patients. Alternatively, as the basal ganglia-thalamocortical circuits have been shown to be essential for motor control (Alexander, DeLong, \& Strick, 1986), alterations in these subcortical structures may also underlie the motor deficits in treated NPC patients, such as dysphagia(McDowell, Corry, Ringash, \& Rischin, 2020).

\section{Limitation}

There are some limitations for this study. First, this was a cross-sectional study. We cannot draw conclusions about the causality of subcortical morphological abnormalities. Future longitudinal studies could address this problem. Second, due to the lack of NPC patients treated with CT alone, we cannot determine the exact role of CT in subcortical morphological abnormalities. Third, the absence of detailed evaluations of psychological status, cognitive functions, and clinical signs and symptoms of patients, undermines the interpretation of our findings.

\section{Conclusion}

In this study, we investigated subcortical morphological alterations in the post-RT and post-CCRT groups compared with the pre-RT group. We found that, compared with the pre-RT group, the post-CCRT group exhibited morphological alterations in the bilateral thalamus, bilateral putamen, left pallidum, and left caudate while the post-RT group was only in the bilateral thalamus, suggesting that there may be an interaction between RT and CT for subcortical structural injury. Our findings provide new insights into the optimization of treatment options for NPC.

\section{Declarations}

\section{Acknowledgements}

The authors would like thank all the study participants for their efforts and enthusiasm for our clinical research. All authors reviewed the manuscript before submission.

\section{Funding}

This study was supported in part by the National Natural Science Foundation of China, Grant/Award Numbers: 82001784, 91959117; The Youth Science Foundation of Xiangya Hospital, Grant/Award Number: 2019Q16. 


\section{Conflict of interest statement}

The authors declare that they have no conflict of interest.

\section{Ethics approval}

This study was approved by the Medical Research Ethics Committee of Xiangya Hospital, Central South University and performed in line with the principles of the Declaration of Helsinki and its later amendments.

\section{Consent to participate}

Written informed consent was obtained from all subjects.

\section{Consent for publication}

Written informed consent for publication was obtained from all participants

\section{Availability of data and material}

The datasets generated during the current study are available from the corresponding author upon reasonable request from a qualified investigator.

\section{Code availability}

Not applicable

\section{Authors' contributions}

Yuanchao Zhang and You-ming Zhang designed and organized the study. You-ming Zhang and Wei-hua Liao contributed to the data collection. Jian-ming Gao and Li Li analyzed the data together. Yuanchao Zhang prepared the figures. Yuanchao Zhang and Feibiao Nan drafted and revised the manuscript. All authors approved the submitted version.

\section{References}

1. Ahles, T. A., \& Saykin, A. J. (2007). Candidate mechanisms for chemotherapy-induced cognitive changes. Nat Rev Cancer, 7(3), 192-201. doi:10.1038/nrc2073

2. Alexander, G. E., DeLong, M. R., \& Strick, P. L. (1986). Parallel organization of functionally segregated circuits linking basal ganglia and cortex. Annual review of neuroscience, 9, 357-381. doi:10.1146/annurev.ne.09.030186.002041

3. Bick, S. K., Patel, S. R., Katnani, H. A., Peled, N., Widge, A., Cash, S. S., \& Eskandar, E. N. (2019). Caudate stimulation enhances learning. Brain, 142(10), 2930-2937. doi:10.1093/brain/awz254 
4. Blanchard, P., Lee, A., Marguet, S., Leclercq, J., Ng, W. T., Ma, J., . . Pignon, J. P. (2015). Chemotherapy and radiotherapy in nasopharyngeal carcinoma: an update of the MAC-NPC metaanalysis. The Lancet Oncology, 16(6), 645-655. doi:10.1016/s1470-2045(15)70126-9

5. Block, M. L., Zecca, L., \& Hong, J. S. (2007). Microglia-mediated neurotoxicity: uncovering the molecular mechanisms. Nat Rev Neurosci, 8(1), 57-69. doi:10.1038/nrn2038

6. Bolkan, S. S., Stujenske, J. M., Parnaudeau, S., Spellman, T. J., Rauffenbart, C., Abbas, A. I., . . . Kellendonk, C. (2017). Thalamic projections sustain prefrontal activity during working memory maintenance. Nat Neurosci, 20(7), 987-996. doi:10.1038/nn.4568

7. Chen, Q., Lv, X., Zhang, S., Lin, J., Song, J., Cao, B., . . Huang, R. (2020). Altered properties of brain white matter structural networks in patients with nasopharyngeal carcinoma after radiotherapy. Brain Imaging Behav. doi:10.1007/s11682-019-00224-2

8. Chen, S. C., Abe, Y., Fang, P. T., Hsieh, Y. J., Yang, Y. I., Lu, T. Y., . . . Hisatsune, T. (2017). Prognosis of Hippocampal Function after Sub-lethal Irradiation Brain Injury in Patients with Nasopharyngeal Carcinoma. Sci Rep, 7(1), 14697. doi:10.1038/s41598-017-13972-2

9. Chua, M. L. K., Wee, J. T. S., Hui, E. P., \& Chan, A. T. C. (2016). Nasopharyngeal carcinoma. The Lancet, 387(10022), 1012-1024. doi:10.1016/s0140-6736(15)00055-0

10. Ding, Z., Zhang, H., Lv, X. F., Xie, F., Liu, L., Qiu, S., . . Shen, D. (2018). Radiation-induced brain structural and functional abnormalities in presymptomatic phase and outcome prediction. Hum Brain Mapp, 39(1), 407-427. doi:10.1002/hbm.23852

11. Doi, T., Fan, Y., Gold, J. I., \& Ding, L. (2020). The caudate nucleus contributes causally to decisions that balance reward and uncertain visual information. Elife, 9. doi:10.7554/eLife.56694

12. Duan, F., Cheng, J., Jiang, J., Chang, J., Zhang, Y., \& Qiu, S. (2016). Whole-brain changes in white matter microstructure after radiotherapy for nasopharyngeal carcinoma: a diffusion tensor imaging study. European Archives of Oto-Rhino-Laryngology, 273(12), 4453-4459. doi:10.1007/s00405-0164127-x

13. Edge, S. B., \& Compton, C. C. (2010). The American Joint Committee on Cancer: the 7th edition of the AJCC cancer staging manual and the future of TNM. Ann Surg Oncol, 17(6), 1471-1474. doi:10.1245/s10434-010-0985-4

14. Genschaft, M., Huebner, T., Plessow, F., Ikonomidou, V. N., Abolmaali, N., Krone, F., ... Smolka, M. N. (2013). Impact of chemotherapy for childhood leukemia on brain morphology and function. PLoS One, 8(11), e78599. doi:10.1371/journal.pone.0078599

15. Guo, Z., Han, L., Yang, Y., He, H., Li, J., Chen, H., . . Lv, X. (2018). Longitudinal brain structural alterations in patients with nasopharyngeal carcinoma early after radiotherapy. Neuroimage Clin, 19, 252-259. doi:10.1016/j.nicl.2018.04.019

16. Hervais-Adelman, A., Egorova, N., \& Golestani, N. (2018). Beyond bilingualism: multilingual experience correlates with caudate volume. Brain Struct Funct, 223(7), 3495-3502.

doi:10.1007/s00429-018-1695-0

Page $11 / 14$ 
17. Hong, J. S., Tian, J., Han, Q. F., \& Ni, Q. Y. (2015). Quality of life of nasopharyngeal cancer survivors in China. Curr Oncol, 22(3), e142-147. doi:10.3747/co.22.2323

18. Hwang, K., Bertolero, M. A., Liu, W. B., \& D'Esposito, M. (2017). The Human Thalamus Is an Integrative Hub for Functional Brain Networks. Journal of Neuroscience, 37(23), 5594-5607. doi:10.1523/jneurosci.0067-17.2017

19. Kafkas, A., Mayes, A. R., \& Montaldi, D. (2020). Thalamic-Medial Temporal Lobe Connectivity Underpins Familiarity Memory. Cereb Cortex, 30(6), 3827-3837. doi:10.1093/cercor/bhz345

20. Leng, X., Fang, P., Lin, H., An, J., Tan, X., Zhang, C., .. Q Qiu, S. (2017). Structural MRI research in patients with nasopharyngeal carcinoma following radiotherapy: A DTI and VBM study. Oncol Lett, 14(5), 6091-6096. doi:10.3892/ol.2017.6968

21. Leng, X., Fang, P., Lin, H., Qin, C., Tan, X., Liang, Y., . . Qiu, S. (2019). Application of a machine learning method to whole brain white matter injury after radiotherapy for nasopharyngeal carcinoma. Cancer Imaging, 19(1), 19. doi:10.1186/s40644-019-0203-y

22. Liddelow, S. A., Guttenplan, K. A., Clarke, L. E., Bennett, F. C., Bohlen, C. J., Schirmer, L., . . Barres, B. A. (2017). Neurotoxic reactive astrocytes are induced by activated microglia. Nature, 541(7638), 481487. doi:10.1038/nature21029

23. Lin, J., Lv, X., Niu, M., Liu, L., Chen, J., Xie, F., .. Huang, R. (2017). Radiation-induced abnormal cortical thickness in patients with nasopharyngeal carcinoma after radiotherapy. Neuroimage Clin, 14, 610-621. doi:10.1016/j.nicl.2017.02.025

24. Lumniczky, K., Szatmari, T., \& Safrany, G. (2017). Ionizing Radiation-Induced Immune and Inflammatory Reactions in the Brain. Front Immunol, 8, 517. doi:10.3389/fimmu.2017.00517

25. Lv, X. F., Zheng, X. L., Zhang, W. D., Liu, L. Z., Zhang, Y. M., Chen, M. Y., \& Li, L. (2014). Radiationinduced changes in normal-appearing gray matter in patients with nasopharyngeal carcinoma: a magnetic resonance imaging voxel-based morphometry study. Neuroradiology, 56(5), 423-430. doi:10.1007/s00234-014-1338-y

26. Makale, M. T., McDonald, C. R., Hattangadi-Gluth, J. A., \& Kesari, S. (2017). Mechanisms of radiotherapy-associated cognitive disability in patients with brain tumours. Nat Rev Neurol, 13(1), 5264. doi:10.1038/nrneurol.2016.185

27. McDowell, L., Corry, J., Ringash, J., \& Rischin, D. (2020). Quality of Life, Toxicity and Unmet Needs in Nasopharyngeal Cancer Survivors. Front Oncol, 10, 930. doi:10.3389/fonc.2020.00930

28. Nakajima, M., \& Halassa, M. M. (2017). Thalamic control of functional cortical connectivity. Curr Opin Neurobiol, 44, 127-131. doi:10.1016/j.conb.2017.04.001

29. Nelson, M. B., Macey, P. M., Harper, R. M., Jacob, E., Patel, S. K., Finlay, J. L., . . Compton, P. (2014). Structural brain alterations in children an average of 5 years after surgery and chemotherapy for brain tumors. J Neurooncol, 119(2), 317-326. doi:10.1007/s11060-014-1480-8

30. Niendam, T. A., Laird, A. R., Ray, K. L., Dean, Y. M., Glahn, D. C., \& Carter, C. S. (2012). Meta-analytic evidence for a superordinate cognitive control network subserving diverse executive functions. Cogn Affect Behav Neurosci, 12(2), 241-268. doi:10.3758/s13415-011-0083-5 
31. Patenaude, B., Smith, S. M., Kennedy, D. N., \& Jenkinson, M. (2011). A Bayesian model of shape and appearance for subcortical brain segmentation. Neuroimage, 56(3), 907-922.

doi:10.1016/j.neuroimage.2011.02.046

32. Prevosto, V., \& Sommer, M. A. (2013). Cognitive control of movement via the cerebellar-recipient thalamus. Front Syst Neurosci, 7, 56. doi:10.3389/fnsys.2013.00056

33. Ren, W. T., Li, Y. X., Wang, K., Gao, L., Yi, J. L., Huang, X. D., . . Dai, J. R. (2019). Cerebral functional abnormalities in patients with nasopharyngeal carcinoma after radiotherapy: an observational magnetic resonance resting-state study. Chin Med J (Engl), 132(13), 1563-1571. doi:10.1097/CM9.0000000000000277

34. Ren, X., Boriero, D., Chaiswing, L., Bondada, S., St. Clair, D. K., \& Butterfield, D. A. (2019). Plausible biochemical mechanisms of chemotherapy-induced cognitive impairment ("chemobrain"), a condition that significantly impairs the quality of life of many cancer survivors. Biochimica et Biophysica Acta (BBA) - Molecular Basis of Disease, 1865(6), 1088-1097. doi:10.1016/j.bbadis.2019.02.007

35. Sherman, S. M. (2016). Thalamus plays a central role in ongoing cortical functioning. Nature Neuroscience, 19(4), 533-541. doi:10.1038/nn.4269

36. Shi, L., Du, F. L., Sun, Z. W., Zhang, L., Chen, Y. Y., Xie, T. M., . . Zhang, M. M. (2018). Radiationinduced gray matter atrophy in patients with nasopharyngeal carcinoma after intensity modulated radiotherapy: a MRI magnetic resonance imaging voxel-based morphometry study. Quant Imaging Med Surg, 8(9), 902-909. doi:10.21037/qims.2018.10.09

37. Simo, M., Vaquero, L., Ripolles, P., Gurtubay-Antolin, A., Jove, J., Navarro, A., . . Rodriguez-Fornells, A. (2016). Longitudinal Brain Changes Associated with Prophylactic Cranial Irradiation in Lung Cancer. J Thorac Oncol, 11(4), 475-486. doi:10.1016/j.jtho.2015.12.110

38. Tang, L. L., Chen, W. Q., Xue, W. Q., He, Y. Q., Zheng, R. S., Zeng, Y. X., \& Jia, W. H. (2016). Global trends in incidence and mortality of nasopharyngeal carcinoma. Cancer Lett, 374(1), 22-30. doi:10.1016/j.canlet.2016.01.040

39. Tang, Y., Luo, D., Rong, X., Shi, X., \& Peng, Y. (2012). Psychological disorders, cognitive dysfunction and quality of life in nasopharyngeal carcinoma patients with radiation-induced brain injury. PLOS One, 7(6), e36529. doi:10.1371/journal.pone.0036529

40. van der Plas, E., Schachar, R. J., Hitzler, J., Crosbie, J., Guger, S. L., Spiegler, B. J., . . Nieman, B. J. (2017). Brain structure, working memory and response inhibition in childhood leukemia survivors. Brain and Behavior, 7(2). doi:10.1002/brb3.621

41. Vinas-Guasch, N., \& Wu, Y. J. (2017). The role of the putamen in language: a meta-analytic connectivity modeling study. Brain Struct Funct, 222(9), 3991-4004. doi:10.1007/s00429-017-1450-y

42. Wang, C., Wang, F., Min, X., Zhang, Q., Shen, L. J., Jiang, Y., \& Yan, J. (2019). Toxicities of chemoradiotherapy and radiotherapy in nasopharyngeal carcinoma: an updated meta-analysis. $J$ Int Med Res, 47(7), 2832-2847. doi:10.1177/0300060519858031 
43. Yan, M., Kumachev, A., Siu, L. L., \& Chan, K. K. (2015). Chemoradiotherapy regimens for locoregionally advanced nasopharyngeal carcinoma: A Bayesian network meta-analysis. Eur J Cancer, 51(12), 1570-1579. doi:10.1016/j.ejca.2015.04.027

44. Yang, Y., Lin, X., Li, J., Han, L., Li, Z., Liu, S., . . Qiu, Y. (2019). Aberrant Brain Activity at Early Delay Stage Post-radiotherapy as a Biomarker for Predicting Neurocognitive Dysfunction Late-Delayed in Patients With Nasopharyngeal Carcinoma. Front Neurol, 10, 752. doi:10.3389/fneur.2019.00752

45. Zhang, Y., Yi, X., Gao, J., Li, L., Liu, L., Qiu, T., . . Liao, W. (2019). Chemotherapy Potentially Facilitates the Occurrence of Radiation Encephalopathy in Patients With Nasopharyngeal Carcinoma Following Radiotherapy: A Multiparametric Magnetic Resonance Imaging Study. Front Oncol, 9, 567. doi:10.3389/fonc.2019.00567

46. Zhang, Y. M., Chen, M. N., Yi, X. P., Li, L., Gao, J. M., Zhang, J. L., . . Zhang, Y. C. (2018). Cortical Surface Area Rather Than Cortical Thickness Potentially Differentiates Radiation Encephalopathy at Early Stage in Patients With Nasopharyngeal Carcinoma. Front Neurosci, 12, 599. doi:10.3389/fnins.2018.00599

47. Zhang, Y. M., Gao, J. M., Zhou, H., Li, L., Liu, L. Z., Han, Z. D., . . Liao, W. H. (2019). Pre-symptomatic local brain activity and functional connectivity alterations in nasopharyngeal carcinoma patients who developed radiation encephalopathy following radiotherapy. Brain Imaging Behav. doi:10.1007/s11682-019-00145-0

48. Zlokovic, B. V. (2008). The blood-brain barrier in health and chronic neurodegenerative disorders. Neuron, 57(2), 178-201. doi:10.1016/j.neuron.2008.01.003

\section{Supplementary Files}

This is a list of supplementary files associated with this preprint. Click to download.

- AuthorChecklist.docx 